\title{
Fuzzy Constrained Probabilistic Inventory Models Depending on Trapezoidal Fuzzy Numbers
}

\author{
Mona F. El-Wakeel ${ }^{1,2}$ and Kholood O. Al-yazidi ${ }^{1}$ \\ ${ }^{1}$ Department of Statistics and Operations Researches, College of Science, King Saud University, P.O. Box 22452, \\ Riyadh 11495, Saudi Arabia \\ ${ }^{2}$ Higher Institute for Computers, Information and Management Technology, Tanta, Egypt
}

Correspondence should be addressed to Mona F. El-Wakeel; melwakeel@ksu.edu.sa

Received 30 March 2016; Accepted 26 July 2016

Academic Editor: Gözde Ulutagay

Copyright (c) 2016 M. F. El-Wakeel and K. O. Al-yazidi. This is an open access article distributed under the Creative Commons Attribution License, which permits unrestricted use, distribution, and reproduction in any medium, provided the original work is properly cited.

\begin{abstract}
We discussed two different cases of the probabilistic continuous review mixture shortage inventory model with varying and constrained expected order cost, when the lead time demand follows some different continuous distributions. The first case is when the total cost components are considered to be crisp values, and the other case is when the costs are considered as trapezoidal fuzzy number. Also, some special cases are deduced. To investigate the proposed model in the crisp case and the fuzzy case, illustrative numerical example is added. From the numerical results we will conclude that Uniform distribution is the best distribution to get the exact solutions, and the exact solutions for fuzzy models are considered more practical and close to the reality of life and get minimum expected total cost less than the crisp models.
\end{abstract}

\section{Introduction}

Inventory system is one of the most diversified fields of applied sciences that are widely used in a variety of areas including operations research, applied probability, computer sciences, management sciences, production system, and telecommunications. More than fifty years ago, the analysis of inventory system has appeared in the reference books and survey papers. Hadley and Whitin [1] are considered one of the first researchers who have discussed the analysis of inventory systems, where they displayed a method for the analysis of the mathematical model for inventory systems. Also, Balkhi and Benkherouf [2] have introduced production lot size inventory model in which products deteriorate at a constant rate and in which demand and production rates are allowed to vary with time. Inventory models may be either deterministic or probabilistic, since the demand of commodity may be deterministic or probabilistic, respectively. These cases were dealt with by Hadley and Whitin [1], Abuo-El-Ata et al. [3], and Vijayan and Kumaran [4].

Some managers allow the shortage in inventory systems; this shortage may be backorder case, lost sales case, and mixture shortage case. Many authors are dealing with inventory problems with various shortage cases where the cost components are considered as crisp values which does not depict the real inventory system fully. For example, constrained probabilistic inventory model with varying order and shortage costs using Lagrangian method has been investigated by Fergany [5]. In addition, constrained probabilistic inventory model with continuous distributions and varying holding cost was discussed by Fergany and El-Saadani [6]. In 2006, several models of continuous distributions for constrained probabilistic lost sales inventory models with varying order cost under holding cost constraint using Lagrangian method by Fergany and El-Wakeel $[7,8]$ were discussed. Recently, El-Wakeel [9] deduced constrained backorders inventory system with varying order cost under holding cost constraint: lead time demand uniformly distributed using Lagrangian method. Also, El-Wakeel and Fergany [10] deduced constrained probabilistic continuous review inventory system with mixture shortage and stochastic lead time demand.

Sometimes, the cost components are considered as fuzzy values, because, in real life, the various physical or chemical 
characteristics may cause an effect on the cost components and then precise values of cost characteristics become difficult to measure as the exact amount of order, holding, and especially shortage cost. Thus, in controlling the inventory system it may allow some flexibility in the cost parameter values in order to treat the uncertainties which always fit the real situations. Since we want to satisfy our requirements for such contradictions, the fuzzy set theory meets these requirements to some extent. In 1965, Zadeh [11] first introduced the fuzzy set theory which studied the intention to accommodate uncertainty in the nonstochastic sense rather than the presence of random variables. Syed and Aziz [12] have examined the fuzzy inventory model without shortages using signed distance method. Kazemi et al. [13] have treated the inventory model with backorders with fuzzy parameters and decision variables. Gawdt [14] presented a mixture continuous review inventory model under varying holding cost constraint when the lead time demand follows Gamma distribution, where the costs were fuzzified as the trapezoidal fuzzy numbers. The continuous review inventory model with mixture shortage under constraint involving crashing cost based on probabilistic triangular fuzzy numbers by Fergany and Gawdt [15] was discussed. A probabilistic periodic review inventory model using Lagrange technique and fuzzy adaptive particle swarm optimization was presented by Fergany et al. [16]. Fuzzy inventory model for deteriorating items with time dependent demand and partial backlogging is established by Kumar and Rajput [17]. Indrajitsingha et al. [18] give fuzzy inventory model with shortages under fully backlogged using signed distance method. Recently, Patel et al. [19] introduced the continuous review inventory model under fuzzy environment without backorder for deteriorating items.

As we found earlier, many authors have studied the inventory models with different assumptions and conditions. These assumptions and conditions are represented in constraints and costs (constant or varying). Therefore, due to the importance of the inventory models we shall propose and study, in this paper, the mixture shortage inventory model with varying order cost under expected order cost constraint and the lead time demand follow Exponential, Laplace, and Uniform distributions. Our goal of studying the inventory models is to minimize the total cost. The order quantity and the reorder point are the policy variables for this model, which minimize the expected annual total cost. We evaluated the optimal order quantity and the reorder point in two cases: first case is when the cost components are considered as crisp values, and the second case is when the cost components are fuzzified as a trapezoidal fuzzy numbers, which is called the fuzzy case. Finally this work is illustrated by numerical example and we will make comparisons of all results and obtain conclusions.

\section{Model Development}

To develop any model of inventory models we need to put some notations and assumptions represented in Notations section.

\subsection{Assumptions}

(1) Consider that continuous review inventory model under order cost constraint and shortages are allowed.

(2) Demand is a continuous random variable with known probability.

(3) The lead time is constant and follows the known distributions.

(4) $\gamma$ is a fraction of unsatisfied demand that will be backordered while the remaining fraction $(1-\gamma)$ is completely lost, where $0 \leq \gamma \leq 1$.

(5) New order with size $(Q)$ is placed when the inventory level drops to a certain level, called the reorder point $(r)$; assume that the system repeats itself in the sense that the inventory position varies between $r$ and $r+Q$ during each cycle.

\section{Model (I): The Mixture Shortage Model Where the Cost Components Are Considered as Crisp Values}

In this section, we consider that the continuous review inventory model with shortage is allowed. Some customers are willing to wait for the new replenishment and the others have no patience; this case is called mixture shortage or partial backorders.

The expected annual total cost consisted of the sum of three components:

$$
\begin{aligned}
E(\text { Total Cost })= & E(\text { order Cost })+E(\text { Holding Cost }) \\
& +E(\text { Shortage Cost }), \\
E(\text { TC }(Q, r))= & E(\mathrm{OC})+E(\mathrm{HC})+E(\mathrm{SC}),
\end{aligned}
$$

where

$$
E(\mathrm{SC})=E(\mathrm{BC})+E(\mathrm{LC})
$$

and we assume the varying order cost function, where the order cost is a decreasing function of the order quantity $Q$. Then, the expected order cost is given by

$$
\begin{aligned}
& E(\mathrm{OC})=c_{o}(\mathrm{Q}) \frac{\bar{D}}{\mathrm{Q}}=c_{o} \mathrm{Q}^{-\beta} \frac{\bar{D}}{\mathrm{Q}}=c_{o} \bar{D} \mathrm{Q}^{-\beta-1}, \\
& E(\mathrm{HC})=c_{h} \bar{H}=c_{h}\left[\frac{\mathrm{Q}}{2}+r-E(x)+(1-\gamma) \bar{S}(r)\right], \\
& E(\mathrm{BC})=\frac{c_{b} \gamma \bar{D}}{\mathrm{Q}} \bar{S}(r) \\
& E(\mathrm{LC})=\frac{c_{l}(1-\gamma) \bar{D}}{\mathrm{Q}} \bar{S}(r) .
\end{aligned}
$$

Our objective is to minimize the expected total costs $[\min E(\mathrm{TC}(Q, r))]$ with varying order cost under the expected order cost constraint which needs to find the 
optimal values of order quantity $Q$ and reorder point $r$. To solve this primal function, let us write it as follows:

$$
\begin{aligned}
E(\mathrm{TC}(Q, r))=c_{o} \bar{D} Q^{-\beta-1} & \\
& +c_{h}\left[\frac{Q}{2}+r-E(x)+(1-\gamma) \bar{S}(r)\right] \\
& +\frac{c_{b} \gamma \bar{D}}{Q} \bar{S}(r)+\frac{c_{l}(1-\gamma) \bar{D}}{Q} \bar{S}(r) \\
= & c_{o} \bar{D} Q^{-\beta-1}+c_{h}\left(\frac{Q}{2}+r-E(x)\right) \\
& +\frac{c_{b} \gamma \bar{D}}{Q} \bar{S}(r) \\
& +\left(c_{h}+\frac{c_{l} \bar{D}}{Q}\right)(1-\gamma) \bar{S}(r)
\end{aligned}
$$

Subject to: $c_{o} \bar{D} Q^{-\beta-1} \leq K$.

We use the Lagrange multiplier technique to get the optimal values $Q^{*}$ and $r^{*}$ which minimize (4) under constraint (5) as follows:

$$
\begin{aligned}
G(Q, r, \lambda)= & c_{o} \bar{D} Q^{-\beta-1}+c_{h}\left(\frac{Q}{2}+r-E(x)\right) \\
& +\frac{c_{b} \gamma \bar{D}}{Q} \bar{S}(r)+\left(c_{h}+\frac{c_{l} \bar{D}}{Q}\right)(1-\gamma) \bar{S}(r) \\
& +\lambda\left(c_{o} \bar{D} Q^{-\beta-1}-K\right) .
\end{aligned}
$$

Putting each of the corresponding first partial derivatives of (6) equal to zero at $Q=Q^{*}$ and $r=r^{*}$, respectively, we get

$$
\begin{aligned}
c_{h} Q^{* 2}+B \bar{D} Q^{*-\beta}-2 A \bar{S}\left(r^{*}\right) & =0, \\
R\left(r^{*}\right) & =\frac{c_{h} Q^{*}}{c_{h}(1-\gamma) Q^{*}+A},
\end{aligned}
$$

where

$$
\begin{aligned}
A & =\bar{D}\left[c_{b} \gamma+c_{l}(1-\gamma)\right], \\
B & =2 c_{o}(-\beta-1)[1+\lambda] .
\end{aligned}
$$

Clearly, it is difficult to find an exact solution of $Q^{*}$ and $r^{*}$ of (7), so we can suppose that the lead time demand follows some distributions.

\subsection{Lead Time Demand Follows Exponential Distribution.} Supposing that the lead time demand follows the Exponential distribution with parameters $v$, then its probability density function is given by

$$
\begin{aligned}
f(x) & =v e^{-v x} ; \quad x \geq 0, v>0 \\
\text { with } E(x) & =\frac{1}{v}, \\
R(r) & =e^{-v r}, \\
\bar{S}(r) & =\frac{1}{v} e^{-v r} .
\end{aligned}
$$

The optimal order quantity and the optimal reorder level which minimize the expected relevant annual total cost can be obtained by substituting (9) into (7). Solving them simultaneously we get

$$
\begin{aligned}
& v c_{h}^{2}(1-\gamma) Q^{* 3}+v c_{h} A Q^{* 2}-2 c_{h} A Q^{*} \\
& \quad+v c_{h}(1-\gamma) B \bar{D} Q^{* 1-\beta}+v A B \bar{D} Q^{*-\beta}=0, \\
& r^{*}=-\frac{1}{v} \ln \left[\frac{c_{h} Q^{*}}{c_{h}(1-\gamma) Q^{*}+A}\right],
\end{aligned}
$$

which give exact solutions for model (I).

3.2. Lead Time Demand Follows Laplace Distribution. If the lead time demand follows the Laplace distribution with parameters $\mu, \theta$, the probability density function will be

$$
f(x)=\frac{1}{2 \theta} e^{-|x-\mu| / \theta} ; \quad-\infty<x<\infty, \theta>0
$$

with $E(x)=\mu$,

$$
\begin{aligned}
& R(r)=\frac{1}{2} e^{-((r-\mu) / \theta)}, \\
& \bar{S}(r)=\frac{\theta}{2} e^{-((r-\mu) / \theta)} .
\end{aligned}
$$

The optimal order quantity and the optimal reorder level which minimize the expected relevant annual total cost can be obtained by substituting (11) into (7), and, solving them simultaneously, we obtain

$$
\begin{aligned}
& c_{h}^{2}(1-\gamma) Q^{* 3}+c_{h} A Q^{* 2}-2 c_{h} \theta A Q^{*} \\
& \quad+c_{h}(1-\gamma) B \bar{D} Q^{* 1-\beta}+A B \bar{D} Q^{*-\beta}=0, \\
& r^{*}=\mu-\theta \ln \left[\frac{2 c_{h} Q^{*}}{c_{h}(1-\gamma) Q^{*}+A}\right],
\end{aligned}
$$

which give exact solutions for model (I).

3.3. Lead Time Demand Follows Uniform Distribution. Similarly, suppose that the lead time demand follows the Uniform distribution over the range from 0 to $b$, that is, $x \sim$ Uniform $(0, b)$; then its probability density function is given by

$$
f(x)=\frac{1}{b} ; \quad 0 \leq x \leq b
$$$$
\text { with } E(x)=\frac{b}{2} \text {, }
$$

$$
\begin{aligned}
& R(r)=1-\frac{r}{b}, \\
& \bar{S}(r)=\frac{1}{2 b}(r-b)^{2} .
\end{aligned}
$$

The optimal order quantity and the optimal reorder level which minimize the expected relevant annual total cost 
can be obtained by substituting (13) into (7). Solving them simultaneously, we find

$$
\begin{aligned}
& c_{h}^{3}(1-\gamma)^{2} Q^{* 4}+2 c_{h}^{2}(1-\gamma) A Q^{* 3} \\
& \quad+c_{h} A\left[A-b c_{h}\right] Q^{* 2}+c_{h}^{2}(1-\gamma)^{2} B \bar{D} Q^{* 2-\beta} \\
& \quad+2 c_{h}(1-\gamma) A B \bar{D} Q^{* 1-\beta}+A^{2} B \bar{D} Q^{*-\beta}=0, \\
& r^{*}=b\left[1-\frac{c_{h} Q^{*}}{c_{h}(1-\gamma) Q^{*}+A}\right],
\end{aligned}
$$

which give exact solutions for model (I).

Thus, the exact solution for constrained continuous review inventory model with mixture shortage and varying order cost can obtained by solving previous equations for each distribution separately at different values of $\beta$ and varying $\lambda$ until the smallest positive value is found such that the constraint holds.

\section{Model $\left(I_{f}\right)$ : The Mixture Shortage Model Where the Cost Components Are Considered as Fuzzy Numbers}

Consider continuous review inventory model similar to model (I), but assuming that the cost components $c_{o}, c_{h}, c_{b}$, and $c_{l}$ are all fuzzy numbers, to control various uncertainties from various physical or chemical characteristics where there may be an effect on the cost components.

We represent these costs by trapezoidal fuzzy numbers as given below:

$$
\begin{aligned}
& \tilde{c}_{o}=\left(c_{o}-\delta_{1}, c_{o}-\delta_{2}, c_{o}+\delta_{3}, c_{o}+\delta_{4}\right), \\
& \tilde{c}_{h}=\left(c_{h}-\delta_{5}, c_{h}-\delta_{6}, c_{h}+\delta_{7}, c_{h}+\delta_{8}\right), \\
& \tilde{c}_{b}=\left(c_{b}-\theta_{1}, c_{b}-\theta_{2}, c_{b}+\theta_{3}, c_{b}+\theta_{4}\right), \\
& \widetilde{c}_{l}=\left(c_{l}-\theta_{5}, c_{l}-\theta_{6}, c_{l}+\theta_{7}, c_{l}+\theta_{8}\right),
\end{aligned}
$$

where $\delta_{i}$ and $\theta_{i}, i=1,2, \ldots, 8$ are arbitrary positive numbers and should satisfy the following constraints:

$$
\begin{aligned}
& c_{o}>\delta_{1}>\delta_{2}, \\
& \delta_{3}<\delta_{4}, \\
& c_{h}>\delta_{5}>\delta_{6}, \\
& \delta_{7}<\delta_{8} .
\end{aligned}
$$

Similarly,

$$
\begin{aligned}
& C_{b}>\theta_{1}>\theta_{2}, \\
& \theta_{3}<\theta_{4}, \\
& \mathcal{c}_{l}>\theta_{5}>\theta_{6}, \\
& \theta_{7}<\theta_{8} .
\end{aligned}
$$

We can represent the order cost as a trapezoidal fuzzy number as shown in Figure 1 and similarly for the remaining costs.

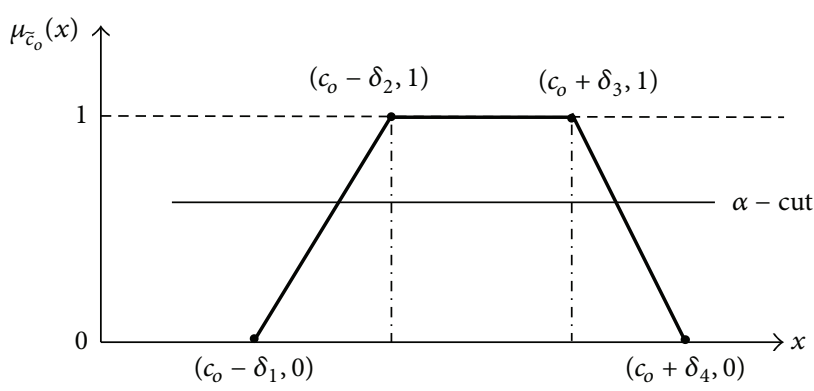

FIgURE 1: Order cost as a trapezoidal fuzzy number.

Note that the membership function of $\widetilde{c}_{o}$ is 1 at points $c_{o}-$ $\delta_{2}$ and $c_{o}+\delta_{3}$, decreases as the point deviates from $c_{o}-\delta_{2}$ and $c_{o}+\delta_{3}$, and reaches zero at the endpoints $c_{o}-\delta_{1}$ and $c_{o}+\delta_{4}$.

The left and right limits of $\alpha-$ cut of $c_{o}, c_{h}, c_{b}$, and $c_{l}$ are given as follows:

$$
\begin{aligned}
& \tilde{c}_{o v}(\alpha)=c_{o}-\delta_{1}+\left(\delta_{1}-\delta_{2}\right) \alpha, \\
& \tilde{c}_{o u}(\alpha)=c_{o}+\delta_{4}-\left(\delta_{4}-\delta_{3}\right) \alpha, \\
& \tilde{c}_{h v}(\alpha)=c_{h}-\delta_{5}+\left(\delta_{5}-\delta_{6}\right) \alpha, \\
& \tilde{c}_{h u}(\alpha)=c_{h}+\delta_{8}-\left(\delta_{8}-\delta_{7}\right) \alpha, \\
& \widetilde{c}_{b v}(\alpha)=c_{b}-\theta_{1}+\left(\theta_{1}-\theta_{2}\right) \alpha, \\
& \widetilde{c}_{b u}(\alpha)=c_{b}+\theta_{4}-\left(\theta_{4}-\theta_{3}\right) \alpha, \\
& \widetilde{c}_{l v}(\alpha)=c_{l}-\theta_{5}+\left(\theta_{5}-\theta_{6}\right) \alpha, \\
& \widetilde{c}_{l u}(\alpha)=c_{l}+\theta_{8}-\left(\theta_{8}-\theta_{7}\right) \alpha .
\end{aligned}
$$

The expected annual total cost for this case under the expected order cost constraint and when all cost components are fuzzy can be expressed as follows:

$$
\begin{aligned}
\widetilde{E}\left(\widetilde{c}_{o}, \widetilde{c}_{h}, \widetilde{c}_{b}, \widetilde{c}_{l}\right)=\widetilde{c}_{o} & \bar{D} Q^{-\beta-1} \\
& +\widetilde{c}_{h}\left[\frac{Q}{2}+r-E(x)+(1-\gamma) \bar{S}(r)\right] \\
& +\frac{\widetilde{c}_{b} \gamma \bar{D}}{Q} \bar{S}(r)+\frac{\widetilde{c}_{l}(1-\gamma) \bar{D}}{Q} \bar{S}(r) \\
= & \widetilde{c}_{o} \bar{D} Q^{-\beta-1}+\widetilde{c}_{h}\left(\frac{Q}{2}+r-E(x)\right) \\
& +\frac{\widetilde{c}_{b} \gamma \bar{D}}{Q} \bar{S}(r) \\
& +\left(\widetilde{c}_{h}+\frac{\widetilde{c}_{l} \bar{D}}{Q}\right)(1-\gamma) \bar{S}(r)
\end{aligned}
$$

Subject to: $\quad \widetilde{c}_{o} \bar{D} Q^{-\beta-1} \leq K$. 
We use the Lagrange multiplier technique to find the optimal values $Q^{*}$ and $r^{*}$ which minimize (19) under constraint (20) as follows:

$$
\begin{aligned}
\widetilde{G}\left(\widetilde{c}_{o}, \widetilde{c}_{h}, \widetilde{c}_{b}, \widetilde{c}_{l}\right)= & \widetilde{c}_{o} \bar{D} Q^{-\beta-1}+\widetilde{c}_{h}\left(\frac{Q}{2}+r-E(x)\right) \\
& +\frac{\widetilde{c}_{b} \gamma \bar{D}}{Q} \bar{S}(r) \\
& +\left(\widetilde{c}_{h}+\frac{\widetilde{c}_{l} \bar{D}}{Q}\right)(1-\gamma) \bar{S}(r) \\
& +\lambda\left(\widetilde{c}_{o} \bar{D} Q^{-\beta-1}-K\right) .
\end{aligned}
$$

We can obtain the form of left and right $\alpha$ - cut of the fuzzified cost function (21), respectively, as follows:

$$
\begin{aligned}
\widetilde{G}\left(\widetilde{c}_{o}, \widetilde{c}_{h}, \widetilde{c}_{b}, \widetilde{c}_{l}\right)_{v}(\alpha)= & \widetilde{c}_{o v} \bar{D} Q^{-\beta-1} \\
& +\widetilde{c}_{h v}\left(\frac{Q}{2}+r-E(x)\right) \\
& +\frac{\widetilde{c}_{b v} \gamma \bar{D}}{Q} \bar{S}(r) \\
& +\left(\widetilde{c}_{h v}+\frac{\widetilde{c}_{l v} \bar{D}}{Q}\right)(1-\gamma) \bar{S}(r) \\
& +\lambda\left(\widetilde{c}_{o v} \bar{D} Q^{-\beta-1}-K\right), \\
\widetilde{G}\left(\widetilde{c}_{o}, \widetilde{c}_{h}, \widetilde{c}_{b}, \widetilde{c}_{l}\right)_{u}(\alpha)= & \widetilde{c}_{o u} \bar{D} Q^{-\beta-1} \\
& +\widetilde{c}_{h u}\left(\frac{Q}{2}+r-E(x)\right) \\
& +\frac{\widetilde{c}_{b u} \gamma \bar{D}}{Q} \bar{S}(r) \\
& +\left(\widetilde{c}_{h u}+\frac{\widetilde{c}_{l u} \bar{D}}{Q}\right)(1-\gamma) \bar{S}(r) \\
& +\lambda\left(\widetilde{c}_{o u} \bar{D} Q^{-\beta-1}-K\right) .
\end{aligned}
$$

Since $\widetilde{G}_{v}(\alpha)$ and $\widetilde{G}_{u}(\alpha)$ exist and are integrable for $\alpha \in[0,1]$, as in $\mathrm{Yao}$ and $\mathrm{Wu}[20]$, we have

$$
d(\widetilde{G}, \widetilde{0})=\frac{1}{2} \int_{0}^{1}\left(\widetilde{G}_{v}(\alpha)+\widetilde{G}_{u}(\alpha)\right) d \alpha
$$

We get the defuzzified value of $\widetilde{G}\left(\widetilde{c}_{o}, \widetilde{c}_{h}, \widetilde{c}_{b}, \widetilde{c}_{l}\right)(\alpha)$ by using (23) for (22) as follows:

$$
\begin{aligned}
d(\widetilde{G}, \widetilde{0})= & A_{1} \bar{D} Q^{-\beta-1}+A_{2}\left(\frac{Q}{2}+r-E(x)\right) \\
& +\frac{A_{3} \gamma \bar{D}}{Q} \bar{S}(r) \\
& +\left(A_{2}+\frac{A_{4} \bar{D}}{Q}\right)(1-\gamma) \bar{S}(r) \\
& +\lambda\left(A_{1} \bar{D} Q^{-\beta-1}-K\right),
\end{aligned}
$$

where

$$
\begin{aligned}
& A_{1}=\frac{\left(4 c_{o}-\delta_{1}-\delta_{2}+\delta_{3}+\delta_{4}\right)}{4}, \\
& A_{2}=\frac{\left(4 c_{h}-\delta_{5}-\delta_{6}+\delta_{7}+\delta_{8}\right)}{4}, \\
& A_{3}=\frac{\left(4 c_{b}-\theta_{1}-\theta_{2}+\theta_{3}+\theta_{4}\right)}{4}, \\
& A_{4}=\frac{\left(4 c_{l}-\theta_{5}-\theta_{6}+\theta_{7}+\theta_{8}\right)}{4} .
\end{aligned}
$$

Similarly, as in model (I), to get the optimal values $Q^{*}$ and $r^{*}$ put each of the corresponding first partial derivatives of (24) equal to zero at $Q=Q^{*}$ and $r=r^{*}$, respectively; we obtain

$$
\begin{aligned}
& \left(2 A_{1}(-\beta-1) \bar{D} Q^{*-\beta}\right)(1+\lambda)+A_{2} Q^{* 2} \\
& \quad-2 A_{3} \gamma \overline{D S}(r)-2 A_{4}(1-\gamma) \overline{D S}(r)=0
\end{aligned}
$$

and the probability of the shortage is

$$
R\left(r^{*}\right)=\frac{A_{2} Q^{*}}{A_{2}(1-\gamma) Q^{*}+A_{3} \gamma \bar{D}+A_{4}(1-\gamma) \bar{D}} .
$$

Clearly, there is no closed form solution of (26) and (27). We can solve these equations by using the same manner as in model (I).

\section{Special Cases}

(1) Letting $\gamma=0, \beta=0$ and $K \rightarrow \infty \Rightarrow C_{o}(Q)=c_{o}$ and $\lambda=0$, thus $A=c_{l} \bar{D}, B=-2 c_{o}$ and hence (7) reduces to

$$
\begin{aligned}
Q^{*} & =\sqrt{\frac{2 \bar{D}\left(c_{o}+c_{l} \bar{s}\left(r^{*}\right)\right)}{c_{h}},} \\
R\left(r^{*}\right) & =\frac{c_{h} Q^{*}}{c_{h} Q^{*}+c_{l} \bar{D}} .
\end{aligned}
$$

This is an unconstrained lost sales continuous review inventory model with constant units of costs, which are the same results as in Hadley and Whitin [1].

(2) Letting $\gamma=1, \beta=0$ and $K \rightarrow \infty \Rightarrow C_{o}(Q)=c_{o}$ and $\lambda=0$, thus $A=c_{b} \bar{D}, B=-2 c_{o}$; thus (7) reduces to

$$
\begin{aligned}
Q^{*} & =\sqrt{\frac{2 \bar{D}\left(c_{o}+c_{b} \bar{s}\left(r^{*}\right)\right)}{c_{h}},} \\
R\left(r^{*}\right) & =\frac{c_{h} Q^{*}}{c_{b} \bar{D}} .
\end{aligned}
$$

This is an unconstrained backorders continuous review inventory model with constant units of costs, which are the same results as in Hadley and Whitin [1]. 
(i) Equations (10) give unconstrained backorders continuous review of inventory model with constant units of cost and the lead time demand follows the Exponential distribution, which are the same results as in Hillier and Lieberman [21].

(ii) Equations (12) give unconstrained backorders continuous review inventory model with constant units of cost and the lead time demand follows the Laplace distribution, which agree with results of Nahmias [22].

(iii) Equations (14) give unconstrained backorders continuous review inventory model with constant units of cost and the lead time demand follows the Uniform distribution, which are the same results as in Fabrycky and Banks [23].

\section{Numerical Example}

Consider an inventory system with the following data:

$\bar{D}=1050$ units per year,

$c_{o}=70$ SR per unit ordered,

$c_{h}=25$ SR per unit per year,

$c_{b}=7$ SR per unit backorder,

$c_{l}=15$ SR per unit lost,

the backorder fraction has the values $\gamma=0.1, \gamma=0.3$, and $\gamma=0.7$,

let $K=140 \mathrm{SR}$,

and take

$$
\begin{aligned}
& \delta_{1}=60, \\
& \delta_{2}=48, \\
& \delta_{3}=10, \\
& \delta_{4}=50, \\
& \delta_{5}=19, \\
& \delta_{6}=10, \\
& \delta_{7}=1, \\
& \delta_{8}=2, \\
& \theta_{1}=6, \\
& \theta_{2}=4, \\
& \theta_{3}=2, \\
& \theta_{4}=4, \\
& \theta_{5}=12, \\
& \theta_{6}=7,
\end{aligned}
$$

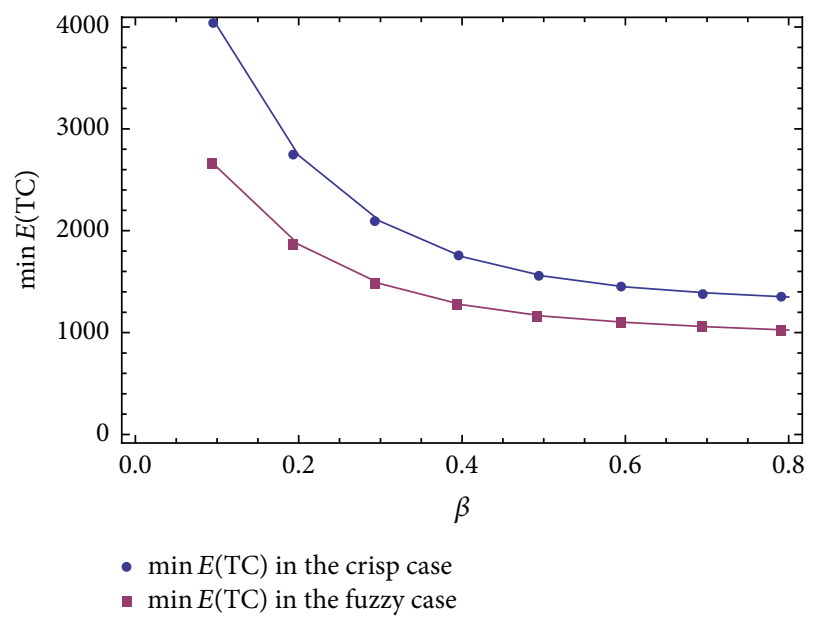

Figure 2: The comparison between the crisp and fuzzy cases for Exponential at $\gamma=0.7$.

$$
\begin{aligned}
& \theta_{7}=1, \\
& \theta_{8}=2 .
\end{aligned}
$$

Determine $Q^{*}$ and $r^{*}$ for both cases of the previous model, when the lead time demand has the following distributions:

(i) Exponential distribution with $v=0.077$ units.

(ii) Laplace distribution with $\mu=13$ and $\theta=10$ units.

(iii) Uniform distribution with $b=26$ units.

Depending on the above data, we can obtain all results by solving the previous deduced equations at different values of $\beta, \lambda$, and $\gamma$ as shown in the Tables 1,2 , and 3 which give the optimal values of $Q^{*}$ and $r^{*}$ that minimize the expected total cost, when the lead time demand follows Exponential, Laplace, and Uniform distribution, respectively, for model (I) and model $\left(\mathrm{I}_{\mathrm{f}}\right)$.

From Table 1 we have that

at $\gamma=0.1$, we will make backorders by $10 \%$ of new orders quantity;

at $\gamma=0.3$, we will make backorders by $30 \%$ of new orders quantity;

at $\gamma=0.7$, we will make backorders by $70 \%$ of new orders quantity.

After comparison of the crisp case and fuzzy case for Exponential distribution, we can deduce that the least $\min E$ (TC) was obtained at $\gamma=0.7$. We can draw the minimum expected total cost for model (I) and model $\left(\mathrm{I}_{\mathrm{f}}\right)$ against $\beta$ for the Exponential distribution at $\gamma=0.7$ as shown in Figure 2 .

From Table 2 we have that

at $\gamma=0.1$, we will make backorders by $10 \%$ of new orders quantity;

at $\gamma=0.3$, we will make backorders by $30 \%$ of new orders quantity; 
TABLE 1: The exact solutions and $\min E(\mathrm{TC})$ for model (I) and model $\left(\mathrm{I}_{\mathrm{f}}\right)$ at Exponential distribution.

\begin{tabular}{|c|c|c|c|c|c|c|c|}
\hline \multirow{2}{*}{$\gamma$} & \multirow{2}{*}{$\beta$} & \multicolumn{3}{|c|}{ Crisp case } & \multicolumn{3}{|c|}{ Fuzzy case } \\
\hline & & $Q^{*}$ & $r^{*}$ & $\min E(\mathrm{TC})$ & $Q^{*}$ & $r^{*}$ & $\min E(\mathrm{TC})$ \\
\hline \multirow{8}{*}{0.1} & 0.1 & 297.092 & 13.8608 & 4199.84 & 250.412 & 15.426 & 2741.44 \\
\hline & 0.2 & 184.893 & 18.4055 & 2910.93 & 158.061 & 20.016 & 1972.09 \\
\hline & 0.3 & 123.76 & 22.6467 & 2252.78 & 107.107 & 24.237 & 1578.8 \\
\hline & 0.4 & 87.6955 & 26.5107 & 1898.63 & 76.6735 & 28.054 & 1367.98 \\
\hline & 0.5 & 65.1132 & 29.9807 & 1703 & 57.4583 & 31.4585 & 1253.06 \\
\hline & 0.6 & 50.1308 & 33.1065 & 1593.97 & 46.7207 & 33.9499 & 1189.84 \\
\hline & 0.7 & 41.8943 & 35.2866 & 1533.94 & 43.0554 & 34.9436 & 1146.05 \\
\hline & 0.8 & 39.0054 & 36.1611 & 1491.79 & 39.9907 & 35.846 & 1112.46 \\
\hline \multirow{8}{*}{0.3} & 0.1 & 297.079 & 11.8026 & 4148.23 & 250.456 & 13.6144 & 2708.31 \\
\hline & 0.2 & 184.905 & 16.4977 & 2863.37 & 158.08 & 18.3589 & 1941.59 \\
\hline & 0.3 & 123.759 & 20.8394 & 2207.59 & 107.132 & 22.6783 & 1550.15 \\
\hline & 0.4 & 87.7063 & 24.768 & 1855.18 & 76.7397 & 26.5535 & 1340.67 \\
\hline & 0.5 & 65.1619 & 28.2749 & 1660.8 & 57.4816 & 30.0074 & 1226.34 \\
\hline & 0.6 & 50.1482 & 31.4365 & 1552.36 & 46.8468 & 32.4964 & 1163.56 \\
\hline & 0.7 & 41.9983 & 33.6078 & 1492.73 & 43.1748 & 33.498 & 1119.93 \\
\hline & 0.8 & 39.1053 & 34.4876 & 1450.74 & 40.1052 & 34.4067 & 1086.48 \\
\hline \multirow{8}{*}{0.7} & 0.1 & 297.118 & 6.33535 & 4012.01 & 250.446 & 8.99977 & 2622.85 \\
\hline & 0.2 & 184.851 & 11.5619 & 2739.35 & 158.081 & 14.2364 & 1865.33 \\
\hline & 0.3 & 123.742 & 16.2344 & 2092.27 & 107.118 & 18.864 & 1479.48 \\
\hline & 0.4 & 87.6963 & 20.3768 & 1745.29 & 76.7206 & 22.9372 & 1273.64 \\
\hline & 0.5 & 65.1605 & 24.0241 & 1554.52 & 57.4204 & 26.5318 & 1161.7 \\
\hline & 0.6 & 50.2168 & 27.266 & 1448.65 & 47.2184 & 28.9822 & 1100.36 \\
\hline & 0.7 & 42.327 & 29.4105 & 1390.22 & 43.5262 & 30.0069 & 1057.21 \\
\hline & 0.8 & 39.4206 & 30.3066 & 1348.72 & 40.4411 & 30.9342 & 1024.17 \\
\hline
\end{tabular}

at $\gamma=0.7$, we will make backorders by $70 \%$ of new orders quantity.

After comparison of the crisp case and fuzzy case for Laplace distribution, we can deduce that the least min $E$ (TC) was obtained at $\gamma=0.7$. We can draw the minimum expected total cost for model $(\mathrm{I})$ and model $\left(\mathrm{I}_{\mathrm{f}}\right)$ against $\beta$ for the Laplace distribution at $\gamma=0.7$ as shown in Figure 3 .

From Table 3 we have that

at $\gamma=0.1$, we will make backorders by $10 \%$ of new orders quantity;

at $\gamma=0.3$, we will make backorders by $30 \%$ of new orders quantity;

at $\gamma=0.7$, we will make backorders by $70 \%$ of new orders quantity.

After comparison of the crisp case and fuzzy case for Uniform distribution, we can deduce that the least min $E$ (TC) was obtained at $\gamma=0.7$. We can draw the minimum expected total cost for model $(\mathrm{I})$ and model $\left(\mathrm{I}_{\mathrm{f}}\right)$ against $\beta$ for the Uniform distribution at $\gamma=0.7$ as shown in Figure 4 .

\section{Conclusion}

In this study we discussed two cases for mixture shortage inventory model under varying order cost constraint when

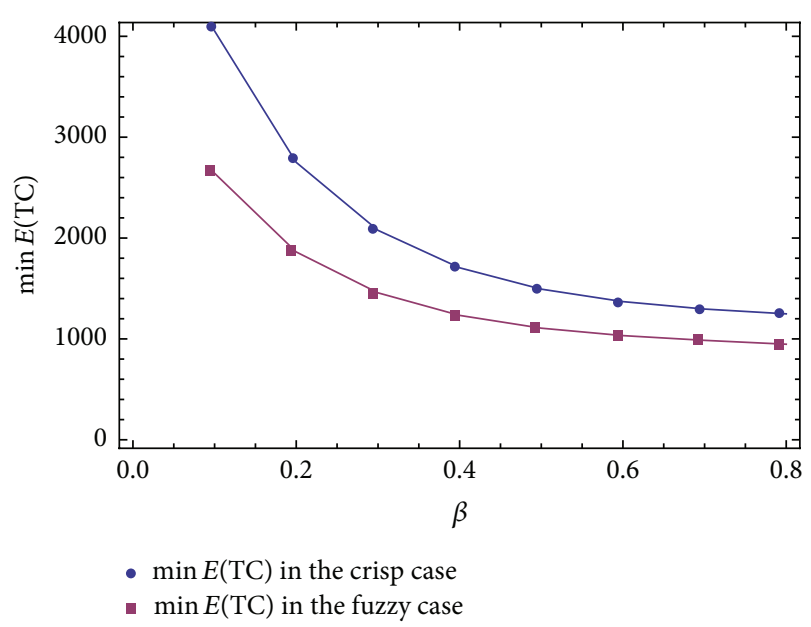

FIgURE 3: The comparison between the crisp and fuzzy cases for Laplace at $\gamma=0.7$.

lead time demand follows Exponential, Laplace, and Uniform distributions. We have evaluated the exact solutions of $Q^{*}$ and $r^{*}$ for each value of $\beta$ and $\lambda^{*}$ which yields our expected order cost constraint and then obtain the minimum expected total cost by using Lagrangian multiplier technique.

By comparing between the minimum expected total cost for model $(\mathrm{I})$ and model $\left(\mathrm{I}_{\mathrm{f}}\right)$ at each distribution, we can 
TABLE 2: The exact solutions and $\min E$ (TC) for model (I) and model $\left(\mathrm{I}_{\mathrm{f}}\right)$ at Laplace distribution.

\begin{tabular}{|c|c|c|c|c|c|c|c|}
\hline \multirow{2}{*}{$\gamma$} & \multirow{2}{*}{$\beta$} & \multicolumn{3}{|c|}{ Crisp case } & \multicolumn{3}{|c|}{ Fuzzy case } \\
\hline & & $Q^{*}$ & $r^{*}$ & $\min E(\mathrm{TC})$ & $Q^{*}$ & $r^{*}$ & $\min E(\mathrm{TC})$ \\
\hline \multirow{8}{*}{0.1} & 0.1 & 297.123 & 16.7406 & 4197.53 & 250.409 & 17.9466 & 2732.79 \\
\hline & 0.2 & 184.844 & 20.2428 & 2881.62 & 158.099 & 21.4789 & 1944.2 \\
\hline & 0.3 & 123.72 & 23.5092 & 2199.23 & 107.093 & 24.7322 & 1532.59 \\
\hline & 0.4 & 87.7213 & 26.4792 & 1823.44 & 76.7474 & 27.6615 & 1305.96 \\
\hline & 0.5 & 65.081 & 29.1581 & 1607.46 & 57.4314 & 30.2959 & 1176.14 \\
\hline & 0.6 & 50.1314 & 31.5604 & 1480.65 & 44.6116 & 32.6421 & 1100.83 \\
\hline & 0.7 & 39.9135 & 33.6954 & 1405.74 & 40.0084 & 33.6658 & 1052.46 \\
\hline & 0.8 & 35.8352 & 34.715 & 1357.91 & 36.8761 & 34.4363 & 1014.82 \\
\hline \multirow{8}{*}{0.3} & 0.1 & 297.09 & 15.1563 & 4157.53 & 250.449 & 16.5519 & 2707.33 \\
\hline & 0.2 & 184.858 & 18.7738 & 2845.06 & 158.053 & 20.2063 & 1920.28 \\
\hline & 0.3 & 123.74 & 22.1162 & 2164.62 & 107.069 & 23.536 & 1510.28 \\
\hline & 0.4 & 87.6956 & 25.141 & 1789.72 & 76.6717 & 26.5228 & 1284.38 \\
\hline & 0.5 & 65.0979 & 27.8493 & 1574.89 & 57.4223 & 29.1839 & 1155.52 \\
\hline & 0.6 & 50.2117 & 30.2628 & 1448.85 & 44.5733 & 31.5604 & 1080.66 \\
\hline & 0.7 & 39.8244 & 32.4508 & 1374.04 & 40.0876 & 32.5659 & 1032.46 \\
\hline & 0.8 & 35.9 & 33.4388 & 1326.43 & 36.9506 & 33.3419 & 994.931 \\
\hline \multirow{8}{*}{0.7} & 0.1 & 297.084 & 10.9477 & 4052.24 & 250.405 & 12.9997 & 2641.24 \\
\hline & 0.2 & 184.853 & 14.9711 & 2749.93 & 158.116 & 17.0285 & 1862.01 \\
\hline & 0.3 & 123.776 & 18.5665 & 2076.28 & 107.095 & 20.5958 & 1456.08 \\
\hline & 0.4 & 87.7171 & 21.7564 & 1705.32 & 76.744 & 23.7273 & 1233.15 \\
\hline & 0.5 & 65.084 & 24.5783 & 1492.99 & 57.4997 & 26.4847 & 1106.02 \\
\hline & 0.6 & 50.1989 & 27.0667 & 1368.85 & 44.5736 & 28.9432 & 1032.24 \\
\hline & 0.7 & 39.9021 & 29.2867 & 1295.45 & 40.3191 & 29.9172 & 984.487 \\
\hline & 0.8 & 36.1029 & 30.2592 & 1248.3 & 37.1679 & 30.7094 & 947.275 \\
\hline
\end{tabular}

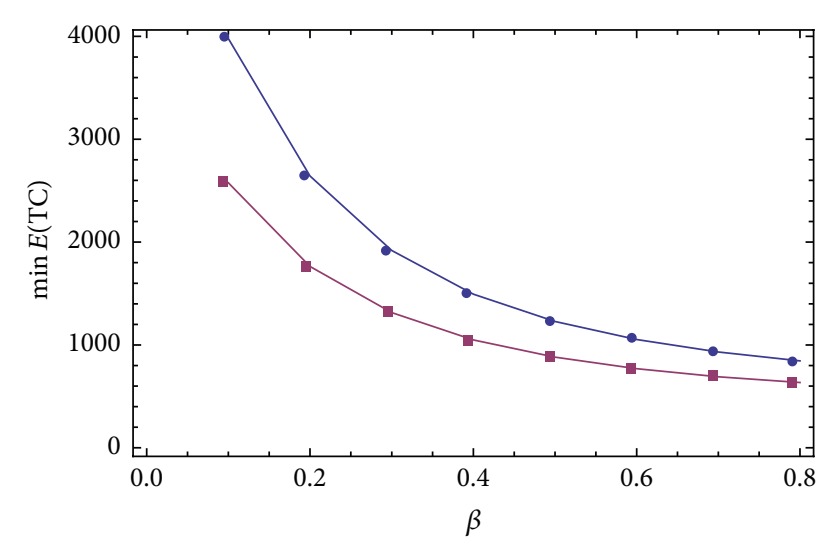

- $\min E(\mathrm{TC})$ in the crisp case

- $\min E(\mathrm{TC})$ in the fuzzy case

FIGURE 4: The comparison between the crisp and fuzzy cases for Uniform at $\gamma=0.7$.

deduce that the least $\min E(\mathrm{TC})$ was obtained when the lead time demand follows Uniform distribution and equals 844.584 SR with order quantity $Q^{*}=32.4596$ and reorder point $r^{*}=23.9138$ for model (I), while the minimum expected annual total cost for model $\left(\mathrm{I}_{\mathrm{f}}\right)$ is $634.709 \mathrm{SR}$ with order quantity $Q^{*}=29.3328$ and reorder point $r^{*}=24.2447$ as shown in Table 3 . This means that we can conclude that the minimum expected total cost in fuzzy case is less than in the crisp case, which indicates that the fuzziness is very close to the actuality of life and gets minimum expected total cost less than the crisp case.

For the results of the numerical example, we note that when $\beta$ increases, $r^{*}$ increases, and thus $Q^{*}$ decreases which indicate that the $\min E(\mathrm{TC})$ decreases.

Also, the different values of $\beta$ lead to changes of $Q^{*}$ in each distribution separately. But in all distributions we note that values of $Q^{*}$ are almost fixed, due to the constraint on the varying order cost. Also, we note that when $\gamma$ increases, $\min E$ (TC) decreases; this indicates that $70 \%$ of the shortages can be met at the lowest possible cost.

Finally, our study in particular provides the ample scope for further research and exploration. For instance, we have considered probabilistic mixture shortage inventory model under varying order cost constraint. This work can be further developed by considering an ample range of different assumptions and conditions represented in constraints and costs (constant or varying), such as varying two costs under two constraints or varying two costs under constraint or varying one cost under two constraints. Also, we can study some of the inventory models with the system multiechelonmultisource. 
TABLE 3: The exact solutions and $\min E(\mathrm{TC})$ for model (I) and model $\left(\mathrm{I}_{\mathrm{f}}\right)$ at Uniform distribution.

\begin{tabular}{|c|c|c|c|c|c|c|c|}
\hline \multirow{2}{*}{$\gamma$} & \multirow{2}{*}{$\beta$} & \multicolumn{3}{|c|}{ Crisp case } & \multicolumn{3}{|c|}{ Fuzzy case } \\
\hline & & $Q^{*}$ & $r^{*}$ & $\min E(\mathrm{TC})$ & $Q^{*}$ & $r^{*}$ & $\min E(\mathrm{TC})$ \\
\hline \multirow{8}{*}{0.1} & 0.1 & 297.124 & 17.0568 & 4067.24 & 250.44 & 18.0722 & 2623.71 \\
\hline & 0.2 & 184.926 & 19.6971 & 2697.71 & 158.084 & 20.4323 & 1791.22 \\
\hline & 0.3 & 123.756 & 21.4539 & 1955.07 & 107.098 & 21.978 & 1333.88 \\
\hline & 0.4 & 87.7442 & 22.6221 & 1519.47 & 76.748 & 22.9994 & 1062.47 \\
\hline & 0.5 & 65.1225 & 23.415 & 1246.58 & 57.4541 & 23.6935 & 890.457 \\
\hline & 0.6 & 50.187 & 23.9661 & 1066.66 & 44.6672 & 24.1744 & 776.304 \\
\hline & 0.7 & 39.8908 & 24.3597 & 942.705 & 35.7373 & 24.5207 & 696.802 \\
\hline & 0.8 & 32.4803 & 24.6502 & 853.887 & 29.23 & 24.7787 & 639.579 \\
\hline \multirow{8}{*}{0.3} & 0.1 & 297.121 & 15.5207 & 4048 & 250.42 & 16.8872 & 2612.58 \\
\hline & 0.2 & 184.862 & 18.7021 & 2684.53 & 158.097 & 19.6747 & 1784.32 \\
\hline & 0.3 & 123.725 & 20.7762 & 1946.25 & 107.066 & 21.4673 & 1328.91 \\
\hline & 0.4 & 87.7476 & 22.1372 & 1513.44 & 76.7256 & 22.6354 & 1058.95 \\
\hline & 0.5 & 65.132 & 23.0538 & 1242.15 & 57.475 & 23.421 & 888.053 \\
\hline & 0.6 & 50.1549 & 23.6892 & 1062.94 & 44.6562 & 23.9647 & 774.318 \\
\hline & 0.7 & 39.8284 & 24.1411 & 939.565 & 35.7041 & 24.3546 & 695.179 \\
\hline & 0.8 & 32.4725 & 24.4703 & 851.602 & 29.2817 & 24.6397 & 638.328 \\
\hline \multirow{8}{*}{0.7} & 0.1 & 297.1 & 10.0378 & 3979.21 & 250.426 & 12.9987 & 2576.66 \\
\hline & 0.2 & 184.862 & 15.3252 & 2642.32 & 158.11 & 17.311 & 1762.56 \\
\hline & 0.3 & 123.72 & 18.5524 & 1918.4 & 107.112 & 19.9169 & 1314.92 \\
\hline & 0.4 & 87.7011 & 20.5852 & 1493.56 & 76.6763 & 21.5568 & 1048.64 \\
\hline & 0.5 & 65.1351 & 21.9128 & 1227.92 & 57.4479 & 22.6277 & 880.563 \\
\hline & 0.6 & 50.1559 & 22.8182 & 1052.06 & 44.6218 & 23.3576 & 768.557 \\
\hline & 0.7 & 39.8823 & 23.4508 & 931.288 & 35.6999 & 23.873 & 690.713 \\
\hline & 0.8 & 32.4596 & 23.9138 & 844.584 & 29.3328 & 24.2447 & 634.709 \\
\hline
\end{tabular}

\section{Notations}

$\begin{array}{ll}\bar{D}: & \begin{array}{l}\text { A random variable denoting the demand } \\ \text { ate per period }\end{array} \\ Q: & \text { A decision variable representing the order } \\ & \text { quantity per cycle } \\ r: & \text { A decision variable representing the } \\ & \text { reorder point } \\ & \text { The lead time between the placement of an } \\ L: & \text { order and its receipt } \\ & \text { The continuous random variable } \\ x: & \text { representing the demand during } L \\ & \text { The probability density function of the } \\ f(x): & \text { lead time demand and }(x) \text { is its } \\ & \text { distribution function } \\ & \text { The probability of the shortage } \\ R(r): & =1-F(r)=\int_{r}^{\infty} f(x) d x \\ \bar{S}(r): & \text { The expected value of shortages per cycle } \\ & =\int_{r}^{\infty}(x-r) f(x) d x \\ c_{o}: & \text { The order cost per unit } \\ C_{o}(Q)=c_{o} Q^{-\beta}: & \text { The varying order cost per cycle } \\ \beta: & \text { A constant real number selected to } \\ & \text { provide the best fit of estimated expected } \\ c_{h}: & \text { cost function } \\ c_{s}: & \text { The holding cost per unit per period } \\ & \text { The shortage cost per unit }\end{array}$

$c_{b}$ : The backorders cost per unit

$c_{l}$ : The lost sales cost per unit

$K$ : The limitation on the expected annual order cost

$\lambda$ : The Lagrangian multiplier.

\section{Competing Interests}

The authors declare that there are no competing interests regarding the publication of this paper.

\section{Acknowledgments}

This research project was supported by a grant from the "Research Center of the Female Scientific and Medical Colleges," Deanship of Scientific Research, King Saud University.

\section{References}

[1] G. Hadley and T. M. Whitin, Analysis of Inventory System, Prentice Hall, Englewood Cliffs, NJ, USA, 1963.

[2] Z. T. Balkhi and L. Benkherouf, "A production lot size inventory model for deteriorating items and arbitrary production and demand rates," European Journal of Operational Research, vol. 92, no. 2, pp. 302-309, 1996. 
[3] M. O. Abuo-El-Ata, H. A. Fergany, and M. F. El-Wakeel, "Probabilistic multi-item inventory model with varying order cost under two restrictions: a geometric programming approach," International Journal of Production Economics, vol. 83, no. 3, pp. 223-231, 2003.

[4] T. Vijayan and M. Kumaran, "Inventory models with a mixture of backorders and lost sales under fuzzy cost," European Journal of Operational Research, vol. 189, no. 1, pp. 105-119, 2008.

[5] H. A. Fergany, Inventory models with demand-dependent units cost [Ph.D. dissertation], Faculty of Science, Tanta University, 1999.

[6] H. A. Fergany and M. E. El-Saadani, "Constrained probabilistic inventory model with continuous distributions and varying holding cost," International Journal of Applied Mathematics, vol. 17, pp. 53-67, 2005.

[7] A. F. Hala and F. E. Mona, "Constrained probabilistic lost sales inventory system with normal distribution and varying order cost," Journal of Mathematics and Statistics, vol. 2, no. 1, pp. 363366, 2006.

[8] H. A. Fergany and M. F. El-Wakeel, "Constrained probabilistic lost sales inventory system with continuous distributions and varying order cost," Journal of Association for the Advancement of Modelling and Simulation Techniques in Enterprises, vol. 27, pp. 3-4, 2006.

[9] M. F. El-Wakeel, "Constrained backorders inventory system with varying order cost: lead time demand uniformly distributed," Journal of King Saud University-Science, vol. 24, no. 3, pp. 285-288, 2012.

[10] M. F. El-Wakeel and H. A. Fergany, "Constrained probabilistic continuous review inventory system with mixture shortage and stochastic lead time demand," Advances in Natural Science, vol. 6, no. 1, pp. 9-13, 2013.

[11] L. A. Zadeh, "Fuzzy sets," Information and Control, vol. 8, pp. 338-353, 1965.

[12] J. K. Syed and L. A. Aziz, "Fuzzy inventory model without shortages using signed distance method," International Journal of Applied Mathematics and Information Sciences, vol. 1, no. 2, pp. 203-209, 2007.

[13] N. Kazemi, E. Ehsani, and M. Y. Jaber, "An inventory model with backorders with fuzzy parameters and decision variables," International Journal of Approximate Reasoning, vol. 51, no. 8, pp. 964-972, 2010.

[14] O. A. Gawdt, Some types of the probabilistic inventory models [Ph.D. thesis], Faculty of Science, Tanta University, 2011.

[15] H. A. Fergany and O. A. Gawdt, "Continuous review inventory model with mixture shortage under constraint involving crashing cost based on probabilistic triangular fuzzy numbers," The Online Journal on Mathematics and Statistics, vol. 2, no. 1, pp. 42-48, 2011.

[16] H. A. Fergany, N. A. El-Hefnawy, and O. M. Hollah, "Probabilistic periodic review $<$ Q_M, N > inventory model using Lagrange technique and fuzzy adaptive particle swarm optimization," Journal of Mathematics and Statistics, vol. 10, no. 3, pp. 368-383, 2014.

[17] S. Kumar and U. S. Rajput, "Fuzzy inventory model for deteriorating items with time dependent demand and partial backlogging," International Journal of Applied Mathematics, vol. 6, no. 3, pp. 496-509, 2015.

[18] S. K. Indrajitsingha, P. N. Samanta, and U. K. Misra, "Fuzzy inventory model with shortages under fully backlogged using signed distance method," International Journal for Research in
Applied Science \& Engineering Technology, vol. 4, pp. 197-203, 2016.

[19] P. D. Patel, A. S. Gor, and P. Bhathawala, "Continuous review inventory model under fuzzy environment without backorder for deteriorating items," International Journal of Applied Research, vol. 2, no. 3, pp. 682-686, 2016.

[20] J.-S. Yao and K. Wu, "Ranking fuzzy numbers based on decomposition principle and signed distance," Fuzzy Sets and Systems, vol. 116, no. 2, pp. 275-288, 2000.

[21] F. S. Hillier and G. J. Lieberman, Introduction to Operations Research, McGraw-Hill, New York, NY, USA, 1995.

[22] S. Nahmias, Production and Operations Analysis, Irwin, Inc, Homewood, Ill, USA, 2nd edition, 1993.

[23] W. J. Fabrycky and J. Banks, Procurement and Inventory Systems: Theory and Analysis, Reinhold Publishing Corporation, New York, NY, USA, 1967. 

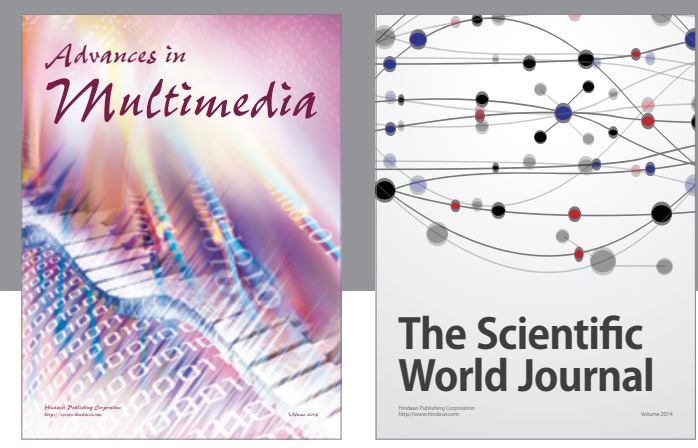

The Scientific World Journal
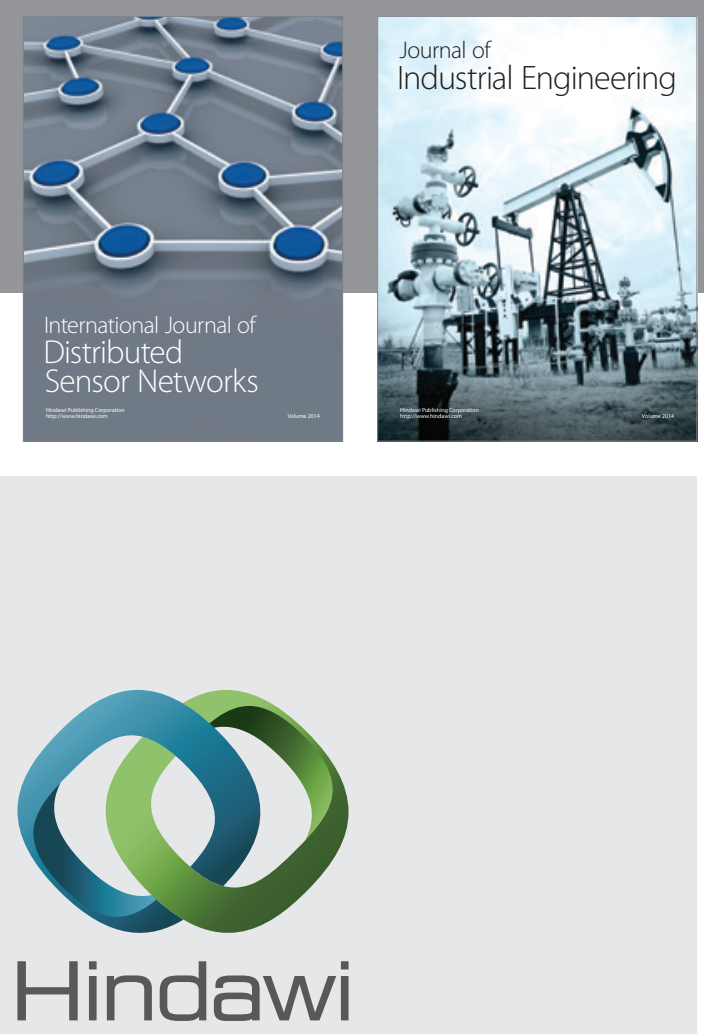

Submit your manuscripts at

http://www.hindawi.com

\section{Computer Networks} and Communications
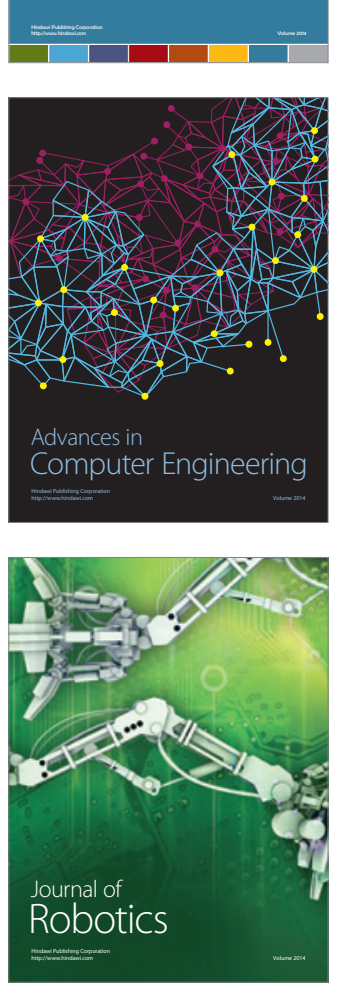
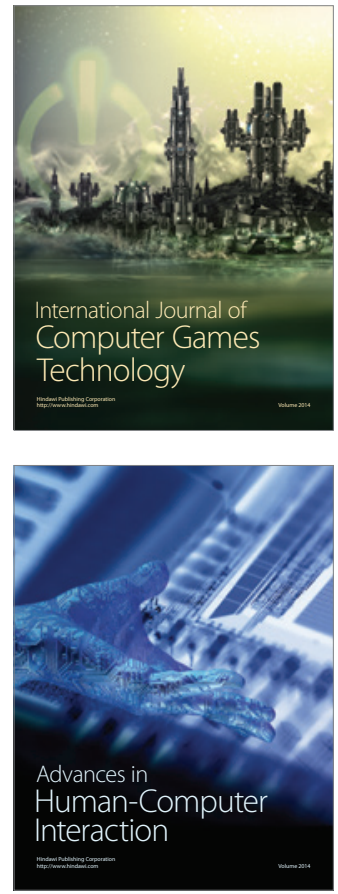
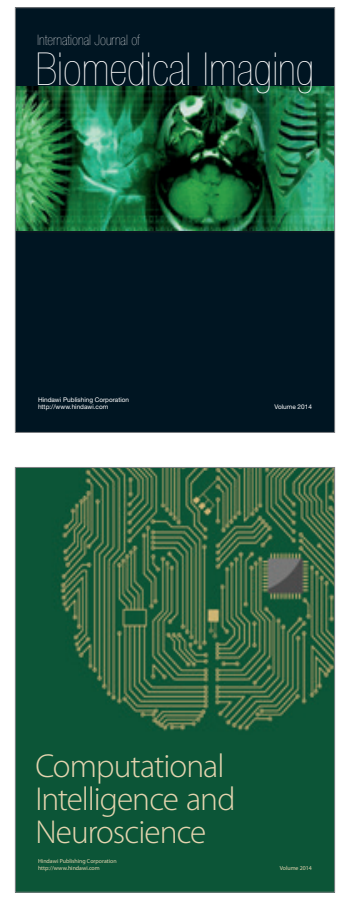
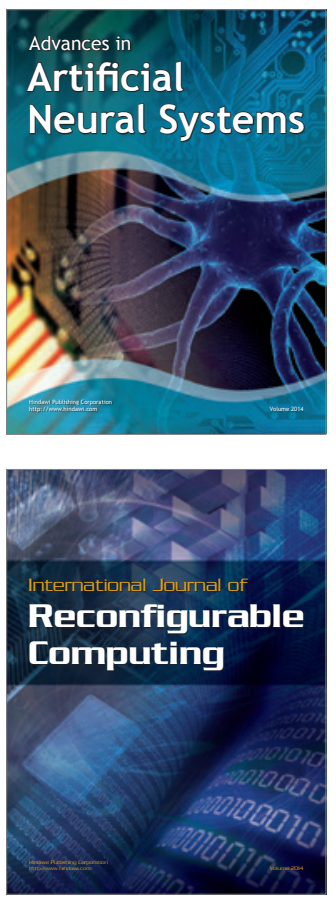
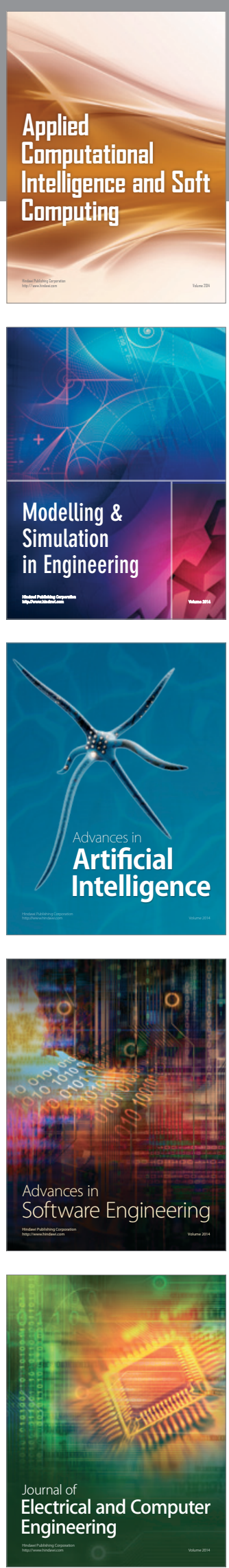